\title{
Construction of B-spline surface from cubic B-spline asymptotic quadrilateral
}

\author{
Hui WANG*, Chun-Gang ZHU* and Cai-Yun LI $^{* *}$ \\ * School of Mathematical Sciences, Dalian University of Technology \\ No.2 Linggong Road, Ganjingzi District, Dalian, 116024, China \\ E-mail: cgzhu@dlut.edu.cn \\ ** School of Mathematics and Physics Science, Dalian University of Technology \\ No.2 Dagong Road, New District of Liaodong Bay, Panjin, 124221, China
}

Received: 13 December 2016; Revised: 25 April 2017; Accepted: 29 May 2017

\begin{abstract}
Asymptote is widely used in astronomy, mechanics, architecture and relevant subjects. In this paper, by analyzing the Frenet frame and the Darboux frame of a curve on the surface, the necessary and sufficient conditions for a quadrilateral boundary being asymptotic of a surface are derived. This quadrilateral is called asymptotic quadrilateral. Given corner data including positions, tangents and curvatures of a cubic B-spline quadrilateral with six control points in each boundary, a family of asymptotic quadrilaterals are constructed after solving the identification conditions of the control points. An optimized one is obtained by minimizing the strain energy of the boundary curves. Then, the transverse tangent vectors along the boundaries of the B-spline surface can be obtained by the asymptotic conditions and the resulting B-spline surface is of bi-quintic degree. Two arrays of control points of the surface along the quadrilateral are obtained from combinations transverse tangent vectors and the boundaries which are elevated from the cubic B-spline curves. For the given inner control points, B-spline surface of bi-quintic degree interpolating the cubic B-spline asymptotic quadrilateral is constructed. The optimized surface is the one with the minimized thin plate spline energy. The method is verified by some representative examples including the boundary curves with lines and inflections. Such interpolation scheme for the construction of the tensor-product B-spline surfaces is compatible with the CAD systems.
\end{abstract}

Keywords : Asymptotic curves, B-spline surface, Interpolation, Quadrilateral, Inflection

\section{Introduction}

Asymptote is one of the most important characteristic curves on surface, which is widely used in astronomy (Contopoulos, 1990), mechanics (Angenent and Velázquez, 1995), architecture (Flöry and Pottmann, 2010), numerical optimization (Wang and Ni, 2008) and so on. Some previous studies of asymptote focused on how to compute or obtain the asymptotic curves on the surface (Wang and Ni, 2008, Lane and MacQueen, 1986, Hartman and Winter, 1951, Kitagawa, 1988). In practice, the reverse problem that how to construct surface interpolating the given curves as asymptotic curves deserves our research, too.

Surfaces interpolating the special curves, such as geodesics and lines of curvature, were widely studied. For instance, Wang et al. (2004) and Li et al. (2011a) constructed parametric surfaces asking a given spatial curve to be a geodesic and a line of curvature, respectively. Furthermore, Kasap et al. (2008) and Li et al. (2013a) separately extended the parametric surfaces into a generalization of surface family interpolating a geodesic curve and a line of curvature. The developable surfaces through Bézier geodesics (Li et at., 2011b) and a given line of curvature (Li et al., 2013b) were also discussed. Notice that the resulting surfaces studied above didn't interpolate the closed boundary curves. Whereas, Farouki, Szafran and Biard studied intensively about this. They constructed a series of Coons surfaces bounded by given closed geodesic curves (Farouki, 2009a, 2009b, 2010) and lines of curvature (Biard, 2010). Triangular Coons patches (Farouki, 2010) and four-sided Bézier patches (Farouki, 2009b) with geodesic boundaries were based on the sufficient 
and necessary conditions for Coons patches interpolating geodesic boundary curves (Farouki, 2009a). Yang and Wang (2015a, 2015b) constructed the tensor-product Bézier surface and B-spline surface of lower degrees through Bézier and B-spline geodesic quadrilateral respectively, which avoided high degrees of the constructed surfaces in (Farouki, 2009b).

Apart from geodesics and lines of curvature, given asymptotic curves to be boundary curves is another interesting topic. Liu and Wang (2013) generalized the result (Wang, et al., 2004) to developable surface through a given asymptote. In architectural design, Flöry and Pottmann (2010) used asymptotic direction, which were estimated from the given point cloud, to construct an initial ruled surface by aligning rulings with asymptotic curves. Shi et al. (2014) presented a method for approximation of a negatively curved surface with a quad mesh guided by the asymptotic directions. However, besides the ruled surfaces, whether there are some other general surfaces to construct freeform surfaces by analyzing the asymptotic direction or interpolating some curves as the asymptotic boundary? Inspired by these applications in architecture, Bayram et al. (2012, 2015, 2016) constructed a family of parametric surfaces interpolating a common asymptote. Atalay and Kasap $(2015,2016)$ and Yüzbasi (2016) identified a surfaces family through an asymptotic curve with Cartan frame, bishop frame and Frenet frame.

Up to now, all these interpolated asymptotic boundary curves studied are not closed. Generally, surface interpolating the closed boundary curve is more interesting for surface modeling in architecture and mechanical design. Motivated by the potential applications in architecture, architectural geometry, and mechanical design, we extend the prior studies to the case that all four boundary curves of a rectangular patch are specified as asymptotic quadrilateral of the constructed surface. We wonder what such surfaces would be like, and whether these surfaces can be constructed just as the surfaces interpolating geodesic quadrilateral or lines of curvature quadrilateral. We identify constraints on boundary quadrilateral, which constitutes the sufficient and necessary conditions for the surface interpolating the asymptotic quadrilateral. To be compatible with the requirements of the commercial CAD systems, we design the tensor product B-spline surface through B-spline asymptotic quadrilateral of lower degree in this paper.

The paper is arranged as follows. Section 2 recalls some notations and results in differential geometry and offers the condition for a regular curve to be an asymptote on the surface. For given corner data, Section 3 identifies the constraints of cubic B-spline asymptotic quadrilateral, followed by the construction of tensor-product B-spline surface interpolating this asymptotic quadrilateral in Section 4. Examples including the boundary curves with lines demonstrate the presented method in Section 5. Additionally, B-spline surfaces interpolating cubic B-spline asymptotic quadrilateral with inflections are also discussed in Section 6. Finally, Section 7 concludes the paper.

\section{Preliminaries}

In this paper, $\dot{\mathbf{r}}(s)$ and $\mathbf{r}^{\prime}(t)$ define the derivatives of $\mathbf{r}$ with respect to arc-length parameter $s$ and arbitrary parameter $t$, respectively. A curve is regular if it has a tangent line at each point of the curve. In the rest of the paper, all curves are assumed to be regular. Next, we recall some basic facts about the Frenet and the Darboux frame from differential geometry (Do Carmo, 1976, Chen, 2011).

The Serret-Frenet frame $(\mathbf{e}(s), \mathbf{n}(s), \mathbf{b}(s))$ is represented by the unit tangent $\mathbf{e}(s)=\dot{\mathbf{r}}(s)$, principal normal $\mathbf{n}(s)=$ $\ddot{\mathbf{r}}(s) /\|\ddot{\mathbf{r}}(s)\|$ and binormal vectors $\mathbf{b}(s)=\mathbf{e}(s) \times \mathbf{n}(s)$ of the space curve at the point $\mathbf{r}(s)$. It satisfies the relations:

$$
\left(\begin{array}{c}
\dot{\mathbf{e}}(s) \\
\dot{\mathbf{n}}(s) \\
\dot{\mathbf{b}}(s)
\end{array}\right)=\left(\begin{array}{ccc}
0 & k(s) & 0 \\
-k(s) & 0 & \tau(s) \\
0 & -\tau(s) & 0
\end{array}\right)\left(\begin{array}{c}
\mathbf{e}(s) \\
\mathbf{n}(s) \\
\mathbf{b}(s)
\end{array}\right),
$$

where the curvature $k(s)$ and torsion $\tau(s)$ of the curve $\mathbf{r}(s)$ are defined by $k(s)=\|\ddot{\mathbf{r}}(s)\|, \quad \tau(s)=\frac{\operatorname{det}(\dot{\mathbf{r}}(s), \ddot{\mathbf{r}}(s), \dot{\mathbf{r}}(s))}{\|\dot{\mathbf{r}}(s)\|^{2}}$. Above frame requires $\ddot{\mathbf{r}}(s) \neq 0$, otherwise, $k(s)=0$ (for some parameter $s$ ) identifies an inflection of the curve.

The Darboux frame $(\mathbf{e}(s), \mathbf{h}(s), \mathbf{N}(s))$ of a space curve $\mathbf{r}(s)=\mathbf{R}(u(s), v(s))$ on the surface $\mathbf{R}(u, v)$ is defined by the unit tangent vector $\mathbf{e}(s)$ of the curve, the unit normal vector $\mathbf{N}(s)=\frac{\mathbf{R}_{u}(u, v) \times \mathbf{R}_{v}(u, v)}{\left\|\mathbf{R}_{u}(u, v) \times \mathbf{R} v(u, v)\right\|}$ of the surface, and $\mathbf{h}(s)=\mathbf{N}(s) \times \mathbf{e}(s)$ at the point $\mathbf{R}(u(s), v(s))=\mathbf{r}(s)$. It's governed by the equation:

$$
\left(\begin{array}{c}
\dot{\mathbf{e}}(s) \\
\dot{\mathbf{h}}(s) \\
\dot{\mathbf{N}}(s)
\end{array}\right)=\left(\begin{array}{ccc}
0 & k_{g}(s) & k_{n}(s) \\
-k_{g}(s) & 0 & -\tau_{g}(s) \\
-k_{n}(s) & \tau_{g}(s) & 0
\end{array}\right)\left(\begin{array}{c}
\mathbf{e}(s) \\
\mathbf{h}(s) \\
\mathbf{N}(s)
\end{array}\right),
$$

where the normal curvature $k_{n}(s)$, the geodesic curvature $k_{g}(s)$, and the geodesic torsion $\tau_{g}(s)$ are defined as $k_{n}(s)=$ $\dot{\mathbf{e}}(s) \cdot \mathbf{N}(s), k_{g}(s)=\dot{\mathbf{e}}(s) \cdot \mathbf{h}(s), \tau_{g}(s)=\dot{\mathbf{N}}(s) \cdot \mathbf{h}(s)$. 
For the parametric curve $\mathbf{r}: t \rightarrow \mathbf{r}(t)$, the Frenet frame is defined by

$$
\left(\begin{array}{c}
\mathbf{e}^{\prime}(t) \\
\mathbf{n}^{\prime}(t) \\
\mathbf{b}^{\prime}(t)
\end{array}\right)=\left\|\mathbf{r}^{\prime}(t)\right\|\left(\begin{array}{ccc}
0 & k(t) & 0 \\
-k(t) & 0 & \tau(t) \\
0 & -\tau(t) & 0
\end{array}\right)\left(\begin{array}{c}
\mathbf{e}(t) \\
\mathbf{n}(t) \\
\mathbf{b}(t)
\end{array}\right)
$$

where $\mathbf{e}(t)=\frac{\mathbf{r}^{\prime}(t)}{\left\|\mathbf{r}^{\prime}(t)\right\|}, \mathbf{b}(t)=\frac{\mathbf{r}^{\prime}(t) \times \mathbf{r}^{\prime \prime}(t)}{\left\|\mathbf{r}^{\prime}(t) \times \mathbf{r}^{\prime \prime}(t)\right\|}, \mathbf{n}(t)=\mathbf{b}(t) \times \mathbf{e}(t)$. The curvature $k(t)$ and torsion $\tau(t)$ of the curve $\mathbf{r}(t)$ are

$$
k(t)=\frac{\left\|\mathbf{r}^{\prime}(t) \times \mathbf{r}^{\prime \prime}(t)\right\|}{\left\|\mathbf{r}^{\prime}(t)\right\|^{3}}, \tau(t)=\frac{\operatorname{det}\left(\mathbf{r}^{\prime}(t), \mathbf{r}^{\prime \prime}(t), \mathbf{r}^{\prime \prime \prime}(t)\right)}{\left\|\mathbf{r}^{\prime}(t) \times \mathbf{r}^{\prime \prime}(t)\right\|^{2}} .
$$

Meanwhile, the Darboux frame $(\mathbf{e}(t), \mathbf{h}(t), \mathbf{N}(t))$ at $\mathbf{r}(t)=\mathbf{R}(u(t), v(t))$ on the surface $\mathbf{R}(u, v)$ is denoted by

$$
\left(\begin{array}{c}
\mathbf{e}^{\prime}(t) \\
\mathbf{h}^{\prime}(t) \\
\mathbf{N}^{\prime}(t)
\end{array}\right)=\left\|u^{\prime} \mathbf{R}_{u}+v^{\prime} \mathbf{R}_{v}\right\|\left(\begin{array}{ccc}
0 & k_{g}(t) & k_{n}(t) \\
-k_{g}(t) & 0 & -\tau_{g}(t) \\
-k_{n}(t) & \tau_{g}(t) & 0
\end{array}\right)\left(\begin{array}{c}
\mathbf{e}(t) \\
\mathbf{h}(t) \\
\mathbf{N}(t)
\end{array}\right)
$$

where $\mathbf{e}=\frac{u^{\prime} \mathbf{R}_{u}+v^{\prime} \mathbf{R}_{v}}{\left\|u^{\prime} \mathbf{R}_{u}+v^{\prime} \mathbf{R}_{v}\right\|}, \mathbf{h}=\frac{\mathbf{R}_{u} \times \mathbf{R}_{v}}{\left\|\mathbf{R}_{u} \times \mathbf{R}_{v}\right\|} \times \mathbf{e}, \mathbf{N}=\frac{\mathbf{R}_{u} \times \mathbf{R}_{v}}{\left\|\mathbf{R}_{u} \times \mathbf{R}_{v}\right\|}$, and $k_{n}(t)=\frac{\mathbf{e}^{\prime}(t) \cdot \mathbf{N}_{(t)}}{\left\|u^{\prime} \mathbf{R}_{u}+v^{\prime} \mathbf{R}_{v}\right\|}, k_{g}(t)=\frac{\mathbf{e}^{\prime}(t) \cdot \mathbf{h}_{(t)}}{\left\|u^{\prime} \mathbf{R}_{u}+v^{\prime} \mathbf{R}_{v}\right\|}, \tau_{g}(t)=\frac{\mathbf{N}^{\prime}(t) \cdot \mathbf{h}_{(t)}}{\left\|u^{\prime} \mathbf{R}_{u}+v^{\prime} \mathbf{R}_{v}\right\|}$.

Definition 1. (Do Carmo, 1976)The curve's asymptotic direction is a direction along which the normal curvature is zero. If the tangent vector $\boldsymbol{e}$ at each point of the curve $\boldsymbol{r}$ is an asymptotic direction of the surface $\boldsymbol{R}(u, v)$, then the curve is an asymptotic curve of the surface.

Therefore, if $\mathbf{r}$ on the surface $\mathbf{R}(u, v)$ is an asymptotic curve, then the normal curvature $k_{n}$ of the curve is identically zero.

Lemma 1. (Chen, 2011) A curve on the surface is an asymptotic curve if and only if it's a line or its osculating surface is its tangent surface.

Lemma 2. A non-linear regular curve $\boldsymbol{r}(t)$ without inflections is an asymptote on the surface $\boldsymbol{R}(u, v)$ if and only if it satisfies the following conditions and all these conditions are equivalent to one another

- the normal curvature of $\boldsymbol{r}(t)$ is identical zero;

- the binormal at each non-inflection point of $\boldsymbol{r}(t)$ is orthogonal to the surface tangent plane at the point $\boldsymbol{R}(u(t), v(t))=$ $\boldsymbol{r}(t)$;

- $\quad$ the osculating plane at each non-inflection point of $\boldsymbol{r}(t)$ is tangent to the surface at the point $\boldsymbol{R}(u(t), v(t))=\boldsymbol{r}(t)$.

Let $p$ be a positive integer and $\Gamma=\left(t_{i}\right)$ be a non-decreasing sequence of real numbers, where $\left\{t_{i}\right\}$ is the set of knots and $\Gamma$ is the knot vector. We denote by $S_{p, \Gamma}$ the linear space spanned by the B-spline basis functions $\left\{N_{i, p, \Gamma}(t)\right\}$ of degree $p$ and with the knot vector $\Gamma$, which satisfy $N_{i, p}(x)=\omega_{i, p}(x) N_{i, p-1}(x)+\left(1-\omega_{i+1, p}(x)\right) N_{i+1, p-1}(x)$ with

$$
\omega_{i, p}(x)=\omega_{i, p, \Gamma}(x)=\left\{\begin{array}{ll}
\frac{x-t_{i}}{t_{i+p-1}-t_{i}}, & \text { if } t_{i}<t_{i+p-1}, \\
0, & \text { otherwise, }
\end{array} \quad \text { and } N_{i, 1}(x)= \begin{cases}1, & \text { if } t_{i} \leq x<t_{i+1}, \\
0, & \text { otherwise }\end{cases}\right.
$$

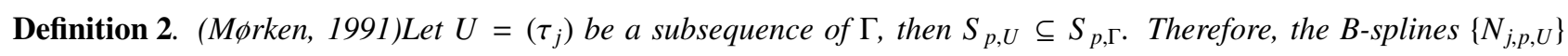
of $S_{p, U}$ are linear combinations of the B-splines $\left\{N_{i, p, \Gamma}\right\}$, that is $N_{j, p, U}=\sum_{i} \alpha_{j, p, U, \Gamma}(i) N_{i, p, \Gamma}$. The coefficients $\left\{\alpha_{j, p, U, \Gamma}\right\}$ are called discrete $B$-splines of degree $p$ on $\Gamma$ with knots $U$, and they satisfy

$$
\alpha_{j, p}(i)=\alpha_{j, p, U, \Gamma}(i)=\omega_{j, p, U}\left(t_{i+p}\right) \alpha_{j, p-1, U, \Gamma}(i)+\left(1-\omega_{j+1, p, U}\left(t_{i+p}\right)\right) \alpha_{j+1, p-1, U, \Gamma}(i),
$$

where $\alpha_{j, 1, U, \Gamma}(i)=N_{j, 1, U}\left(t_{i}\right)$ and $\omega_{j, p, U}(t)=\omega_{j, p}(t)$.

\section{Cubic B-spline asymptotic quadrilateral}

We aim to construct a B-spline patch whose boundary quadrilateral is asymptotic. This boundary quadrilateral is called asymptotic quadrilateral. In order to achieve this goal, we need to consider both the local corner conditions and global boundary conditions. In fact, we can firstly construct the asymptotic quadrilateral under the local condition, then use this quadrilateral to produce the interpolating surface under the global condition.

Before constructing cubic B-spline asymptotic quadrilateral, we analyze the conditions of two asymptotic curves intersecting on the same surface at first. 


\subsection{Conditions of asymptotic curves intersecting on the same surface}

For an asymptotic curve of a surface, we have $k=\sigma k_{g}, \sigma= \pm 1$ due to $k^{2}=k_{g}^{2}+k_{n}^{2}$. From Eq. (1) and Eq. (2), it's easy to get that $\mathbf{h}=\sigma \mathbf{n}$ and $\mathbf{N}=\sigma \mathbf{b}$ for the arc-length parameter. For the general parameter, we have the same results from Eq. (3) and Eq. (5). Therefore, the Frenet and the Darboux frames agree modulo signs for both kinds of parameters. It's easy to deduce the following lemma.

Lemma 3. For an arc-length parametric regular curve $\boldsymbol{r}(s)$ on a surface $\boldsymbol{R}(u, v)$, setting $\boldsymbol{N}(s)=\sigma \boldsymbol{b}(s)$ with $\sigma= \pm 1$, we have $\boldsymbol{h}(s)=\sigma \boldsymbol{n}(s), k_{n}(s)=0, k(s)=\sigma k_{g}(s), \tau_{g}(s)=-\tau(s)$.

Proposition 1. If two arc-length parametric asymptotic curves $\boldsymbol{r}_{1}(s)$ and $\boldsymbol{r}_{2}(s)$ on the same surface $\boldsymbol{R}(u, v)$ intersect at an non-inflection point $\boldsymbol{P}=\boldsymbol{r}_{1}\left(s_{1}\right)=\boldsymbol{r}_{2}\left(s_{2}\right)$ satisfying $\boldsymbol{N}_{P}=\sigma_{1} \boldsymbol{b}_{1}\left(s_{1}\right)=\sigma_{2} \boldsymbol{b}_{2}\left(s_{2}\right)$ with $\sigma_{1}, \sigma_{2}= \pm 1$, then $\tau_{1}\left(s_{1}\right)=-\tau_{2}\left(s_{2}\right)$, where $\boldsymbol{N}_{P}$ is the surface normal, $\boldsymbol{b}_{1}\left(s_{1}\right), \tau_{1}\left(s_{1}\right)$ and $\boldsymbol{b}_{2}\left(s_{2}\right), \tau_{2}\left(s_{2}\right)$ are respectively the binormal vectors and torsion of $\boldsymbol{r}_{1}\left(s_{1}\right)$ and $\boldsymbol{r}_{2}\left(s_{2}\right)$ at the point $\boldsymbol{P}$.

Proof 1. From the above lemma, $\tau_{g 1}(s)=-\tau_{1}(s)$ and $\tau_{g 2}(s)=-\tau_{2}(s)$, where $\tau_{g 1}(s), \tau_{g 2}(s)$ are the geodesic torsion of $\boldsymbol{r}_{1}(s)$ and $\boldsymbol{r}_{2}(s)$. From (Farouki, et al. 2009a), $\sin \left(\alpha_{2}-\alpha_{1}\right)\left[\tau_{g 1}\left(\alpha_{1}\right)+\tau_{g 2}\left(\alpha_{2}\right)\right]=\cos \left(\alpha_{2}-\alpha_{1}\right)\left[k_{n 1}\left(\alpha_{1}\right)-k_{n 2}\left(\alpha_{2}\right)\right]$, where $\alpha_{1}, \alpha_{2}$ specify two directions in the surface tangent plane, then $\sin \left(\alpha_{2}-\alpha_{1}\right) \neq 0$ induces $\tau_{1}\left(s_{1}\right)=-\tau_{2}\left(s_{2}\right)$.

Remark 1. Note that we can get the same results of Lemma 3 and Proposition 1 for the curves with arbitrary parameter. Then for the following discussions, the parameters of the curve $\boldsymbol{r}$ will be omitted if no confusion arises.

If we generalize two asymptotic crossing curves to a quadrilateral, we can have the following necessary conditions for the quadrilateral being asymptotic. In fact, if the boundary quadrilateral doesn't satisfy these conditions, then the boundary curves of the interpolating surface are not asymptotic.

Proposition 2. Four curves $\boldsymbol{r}_{i}(t), i=1, \cdots, 4$, (as shown in Fig. 1) are free of inflections as boundary asymptotes of a surface $\boldsymbol{R}(u, v)$ if and only if these four curves satisfy the following three constraints:

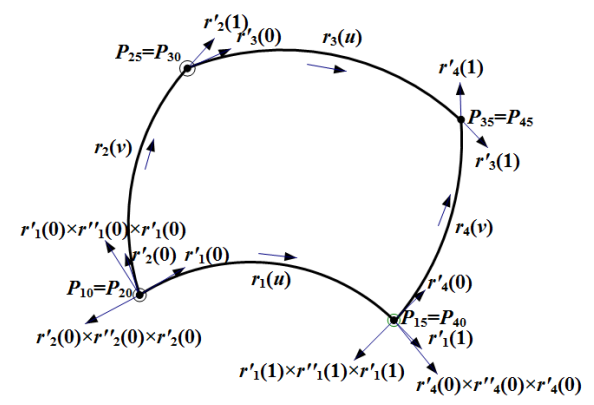

Fig. 1 Quadrilateral with vectors at four corners.

(C1) rectifying constraints: the binormal vectors of the four curves at four corners satisfy the modulo sign, that is

$$
\begin{aligned}
& \boldsymbol{N}_{12}=\sigma_{1}(0) \boldsymbol{b}_{1}(0)=\sigma_{2}(0) \boldsymbol{b}_{2}(0), \boldsymbol{N}_{14}=\sigma_{1}(1) \boldsymbol{b}_{1}(1)=\sigma_{4}(0) \boldsymbol{b}_{4}(0), \\
& \boldsymbol{N}_{23}=\sigma_{2}(1) \boldsymbol{b}_{2}(1)=\sigma_{3}(0) \boldsymbol{b}_{3}(0), \boldsymbol{N}_{34}=\sigma_{3}(1) \boldsymbol{b}_{3}(1)=\sigma_{4}(1) \boldsymbol{b}_{4}(1),
\end{aligned}
$$

where $\boldsymbol{N}_{12}, \boldsymbol{N}_{14}, \boldsymbol{N}_{23}, \boldsymbol{N}_{34}$ are unit normal vectors of the surface at four corners and $\sigma_{i}(j)= \pm 1, i=1, \cdots, 4, j=0,1$.

(C2) corner asymptotic crossing constraints:

$\tau_{1}(0)=-\tau_{2}(0), \tau_{1}(1)=-\tau_{4}(0), \tau_{2}(1)=-\tau_{3}(0), \tau_{3}(1)=-\tau_{4}(1)$.

(C3) boundary normal orientation constraint: boundary normal vectors $\boldsymbol{N}_{i}(t)$ are continuous and satisfy $\boldsymbol{N}_{i}(t)=$ $\pm \boldsymbol{b}_{i}(t), i=1, \cdots, 4$.

Here, $(C 1)$ and $(C 2)$ are the local corner conditions and $(C 3)$ is the global boundary condition. 


\subsection{Constraints for cubic B-spline asymptotic quadrilateral}

In order to construct the cubic B-spline asymptotic quadrilateral, we need to obtain the boundary control points which are determined by the above conditions with the given corner positions, tangent vectors and curvatures. More knots of the boundary B-spline curve means more control points and then more free parameters left to be solved. We find that it's a better choice to satisfy the constraints and solve easily, if we choose the knot vector $\mathbf{U}_{i}=\left\{u_{i 0}, u_{i 1}, u_{i 2}, u_{i 3}, u_{i 4}, u_{i 5}, u_{i 6}, u_{i 7}, u_{i 8}, u_{i 9}\right\}$ with $u_{i j}=0(j=0, \cdots, 3), u_{i j}=1(j=6, \cdots, 9)$ and $0<u_{i 4}<u_{i 5}<1$, which leads to six control points to be solved at each boundary.

Suppose that the four boundary curves with free of inflections are cubic B-spline curves

$$
\mathbf{r}_{i}(u)=\sum_{j=0}^{5} \mathbf{P}_{i j} N_{j, 3}(u),(i=1,3), \mathbf{r}_{i}(v)=\sum_{j=0}^{5} \mathbf{P}_{i j} N_{j, 3}(v),(i=2,4),
$$

where $\mathbf{P}_{i j}$ are control points, and $N_{j, 3}(u), N_{j, 3}(v)$ are B-spline bases defined over $\mathbf{U}_{i}$. Thus corner data meet

$$
\begin{aligned}
& \mathbf{r}_{1}(0)=\mathbf{P}_{10}=\mathbf{P}_{20}=\mathbf{r}_{2}(0), \mathbf{r}_{1}(1)=\mathbf{P}_{15}=\mathbf{P}_{40}=\mathbf{r}_{4}(0), \\
& \mathbf{r}_{2}(1)=\mathbf{P}_{25}=\mathbf{P}_{30}=\mathbf{r}_{3}(0), \mathbf{r}_{3}(1)=\mathbf{P}_{35}=\mathbf{P}_{45}=\mathbf{r}_{4}(1) .
\end{aligned}
$$

Given the value of Eq. (9), points $\mathbf{P}_{11}, \mathbf{P}_{14}, \mathbf{P}_{21}, \mathbf{P}_{24}, \mathbf{P}_{31}, \mathbf{P}_{34}, \mathbf{P}_{41}, \mathbf{P}_{44}$ and curvatures $k_{i}(j), i=1, \cdots, 4, j=0,1$, the rest of control points of B-spline curves $\mathbf{P}_{12}, \mathbf{P}_{13}, \mathbf{P}_{22}, \mathbf{P}_{23}, \mathbf{P}_{32}, \mathbf{P}_{33}, \mathbf{P}_{42}, \mathbf{P}_{43}$ can be obtained from the following constraints.

(a) Rectifying constraints.

From the rectifying constraints in Eq. (6), the unit normal vector $\mathbf{N}_{12}=\frac{\mathbf{r}_{1}^{\prime}(0) \times \mathbf{r}_{2}^{\prime}(0)}{\left\|\mathbf{r}_{1}^{\prime}(0) \times \mathbf{r}_{2}^{\prime}(0)\right\|}$ of the surface is parallel to the unit binormal vectors $\mathbf{b}_{1}(0)=\frac{\mathbf{r}_{1}^{\prime}(0) \times \mathbf{r}_{1}^{\prime \prime}(0)}{\left\|\mathbf{r}_{1}^{\prime}(0) \times \mathbf{r}_{1}^{\prime \prime}(0)\right\|}$ and $\mathbf{b}_{2}(0)=\frac{\mathbf{r}_{2}^{\prime}(0) \times \mathbf{r}_{2}^{\prime \prime}(0)}{\left\|\mathbf{r}_{2}^{\prime}(0) \times \mathbf{r}_{2}^{\prime \prime}(0)\right\|}$, then both $\mathbf{r}_{1}^{\prime \prime}(0)$ and $\mathbf{r}_{2}^{\prime \prime}(0)$ are located in the tangent plane $\Pi_{12}$ of the surface at the point $\mathbf{P}_{10}$. Therefore, points $\mathbf{P}_{10}, \mathbf{P}_{11}, \mathbf{P}_{12}, \mathbf{P}_{21}, \mathbf{P}_{22}$ are coplanar. Let $\Delta \mathbf{P}_{i j}=\mathbf{P}_{i j+1}-\mathbf{P}_{i j}$, $\Delta^{k} \mathbf{P}_{i j}=\Delta^{k-1} \mathbf{P}_{i j+1}-\Delta^{k-1} \mathbf{P}_{i j}, k=2,3$, and $i, j=1, \cdots, 4$. Then $\mathbf{P}_{12}$ and $\mathbf{P}_{22}$ can be represented as

$$
\mathbf{P}_{12}=\mathbf{P}_{10}+\lambda_{12}\left(\Delta \mathbf{P}_{10}\right)+\mu_{12}\left(\Delta \mathbf{P}_{20}\right), \mathbf{P}_{22}=\mathbf{P}_{20}+\lambda_{22}\left(\Delta \mathbf{P}_{10}\right)+\mu_{22}\left(\Delta \mathbf{P}_{20}\right) .
$$

Similarly, $\mathbf{P}_{32}, \mathbf{P}_{23}, \mathbf{P}_{13}, \mathbf{P}_{42}, \mathbf{P}_{33}, \mathbf{P}_{43}$ are represented as:

$$
\begin{aligned}
& \mathbf{P}_{32}=\mathbf{P}_{30}+\lambda_{32}\left(\Delta \mathbf{P}_{24}\right)+\mu_{32}\left(\Delta \mathbf{P}_{30}\right), \mathbf{P}_{23}=\mathbf{P}_{25}+\lambda_{23}\left(\Delta \mathbf{P}_{24}\right)+\mu_{23}\left(\Delta \mathbf{P}_{30}\right), \\
& \mathbf{P}_{13}=\mathbf{P}_{15}+\lambda_{13}\left(\Delta \mathbf{P}_{40}\right)+\mu_{13}\left(\Delta \mathbf{P}_{14}\right), \mathbf{P}_{42}=\mathbf{P}_{40}+\lambda_{42}\left(\Delta \mathbf{P}_{40}\right)+\mu_{42}\left(\Delta \mathbf{P}_{14}\right), \\
& \mathbf{P}_{33}=\mathbf{P}_{35}+\lambda_{33}\left(\Delta \mathbf{P}_{34}\right)+\mu_{33}\left(\Delta \mathbf{P}_{44}\right), \mathbf{P}_{43}=\mathbf{P}_{45}+\lambda_{43}\left(\Delta \mathbf{P}_{34}\right)+\mu_{43}\left(\Delta \mathbf{P}_{44}\right),
\end{aligned}
$$

where $\lambda_{i j}, \mu_{i j}, i=1, \cdots, 4, j=2,3$ are real.

Due to the curvature expression in Eq. (4), we have

$$
k_{1}(0)=\frac{2 u_{14}}{3 u_{15}} \frac{\left\|\left(\Delta \mathbf{P}_{10}\right) \times\left(\Delta^{2} \mathbf{P}_{10}\right)\right\|}{\left\|\Delta \mathbf{P}_{10}\right\|^{3}}, k_{1}(1)=\frac{2\left(1-u_{15}\right)}{3\left(1-u_{14}\right)} \frac{\left\|\left(\Delta \mathbf{P}_{14}\right) \times\left(\Delta^{2} \mathbf{P}_{13}\right)\right\|}{\left\|\Delta \mathbf{P}_{14}\right\|^{3}},
$$

and the other six curvature equations, so that $\lambda_{13}, \mu_{12}, \lambda_{22}, \mu_{23}, \lambda_{32}, \mu_{33}, \lambda_{43}, \mu_{42}$ can be represented by $u_{i 4}, u_{i 5}(i=$ $1, \cdots, 4)$.

(b) Corner asymptotic crossing constraints.

From the torsion constraints in Eq. (7) at four corners, the torsion expression in Eq. (4) leads to

$$
\begin{gathered}
u_{14} \cdot \frac{\operatorname{det}\left(\Delta \mathbf{P}_{10}, \Delta^{2} \mathbf{P}_{10}, \Delta^{3} \mathbf{P}_{10}\right)}{\left\|\left(\Delta \mathbf{P}_{10}\right) \times\left(\Delta^{2} \mathbf{P}_{10}\right)\right\|^{2}}=-u_{24} \cdot \frac{\operatorname{det}\left(\Delta \mathbf{P}_{20}, \Delta^{2} \mathbf{P}_{20}, \Delta^{3} \mathbf{P}_{20}\right)}{\left\|\left(\Delta \mathbf{P}_{20}\right) \times\left(\Delta^{2} \mathbf{P}_{20}\right)\right\|^{2}}, \\
\left(1-u_{15}\right) \cdot \frac{\operatorname{det}\left(\Delta \mathbf{P}_{14}, \Delta^{2} \mathbf{P}_{13}, \Delta^{3} \mathbf{P}_{12}\right)}{\left\|\left(\Delta \mathbf{P}_{14}\right) \times\left(\Delta^{2} \mathbf{P}_{13}\right)\right\|^{2}}=-u_{44} \cdot \frac{\operatorname{det}\left(\Delta \mathbf{P}_{40}, \Delta^{2} \mathbf{P}_{40}, \Delta^{3} \mathbf{P}_{40}\right)}{\left\|\left(\Delta \mathbf{P}_{40}\right) \times\left(\Delta^{2} \mathbf{P}_{40}\right)\right\|^{2}}, \\
\left(1-u_{25}\right) \cdot \frac{\operatorname{det}\left(\Delta \mathbf{P}_{24}, \Delta^{2} \mathbf{P}_{23}, \Delta^{3} \mathbf{P}_{22}\right)}{\left\|\left(\Delta \mathbf{P}_{24}\right) \times\left(\Delta^{2} \mathbf{P}_{23}\right)\right\|^{2}}=-u_{34} \cdot \frac{\operatorname{det}\left(\Delta \mathbf{P}_{30}, \Delta^{2} \mathbf{P}_{30}, \Delta^{3} \mathbf{P}_{30}\right)}{\left\|\left(\Delta \mathbf{P}_{30}\right) \times\left(\Delta^{2} \mathbf{P}_{30}\right)\right\|^{2}}, \\
\left(1-u_{35}\right) \cdot \frac{\operatorname{det}\left(\Delta \mathbf{P}_{34}, \Delta^{2} \mathbf{P}_{33}, \Delta^{3} \mathbf{P}_{32}\right)}{\left\|\left(\Delta \mathbf{P}_{34}\right) \times\left(\Delta^{2} \mathbf{P}_{33}\right)\right\|^{2}}=-\left(1-u_{45}\right) \cdot \frac{\operatorname{det}\left(\Delta \mathbf{P}_{44}, \Delta^{2} \mathbf{P}_{43}, \Delta^{3} \mathbf{P}_{42}\right)}{\left\|\left(\Delta \mathbf{P}_{44}\right) \times\left(\Delta^{2} \mathbf{P}_{43}\right)\right\|^{2}},
\end{gathered}
$$


such that $\mu_{13}, \mu_{22}, \mu_{32}, \mu_{43}$ can also be represented by $u_{i 4}, u_{i 5}$. If given $u_{i 4}, u_{i 5}(i=1, \cdots, 4)$, then $\lambda_{12}, \lambda_{23}, \lambda_{33}, \lambda_{42}$ can be obtained by minimizing the strain energy

$$
\int_{0}^{1}\left(\left\|\mathbf{r}_{1}^{\prime \prime}(u)\right\|^{2}+\left\|\mathbf{r}_{3}^{\prime \prime}(u)\right\|^{2}\right) d u+\int_{0}^{1}\left(\left\|\mathbf{r}_{2}^{\prime \prime}(v)\right\|^{2}+\left\|\mathbf{r}_{4}^{\prime \prime}(v)\right\|^{2}\right) d v .
$$

Hence, the eight unknown points $\mathbf{P}_{12}, \mathbf{P}_{13}, \mathbf{P}_{22}, \mathbf{P}_{23}, \mathbf{P}_{32}, \mathbf{P}_{33}, \mathbf{P}_{42}, \mathbf{P}_{43}$ are determined.

(c) Boundary normal orientation constraint.

Along the regular boundary curves $\mathbf{r}_{i}$, if the unit normal $\mathbf{N}_{i}$ of the surface and binormal $\mathbf{b}_{i}(t), i=1, \cdots, 4$, are parallel and continuous, then the number of reversals at four corners is even. That is

$$
\prod_{i, j} \sigma_{i}(j)=1,(i=1, \cdots, 4, j=0,1)
$$

Compatible with the constraint in Eq. (10), the signs of $\sigma_{i}(j)(i=1, \cdots, 4, j=0,1)$ are chosen based on the equations in Eq. (6).

\section{B-spline surface interpolating B-spline asymptotic quadrilateral}

Constructing the interpolating surface needs to satisfy the global boundary condition, that is, the boundary normal vectors $\mathbf{N}_{i}(t)$ are continuous and satisfy $\mathbf{N}_{i}(t)= \pm \mathbf{b}_{i}(t), i=1, \cdots, 4$. Based on the Darboux frame, the transverse tangent vectors $\mathbf{T}_{i}$ along the boundary curves $\mathbf{r}_{i}(t)$ of the B-spline surface $\mathbf{R}(u, v)$ are defined as

$$
\mathbf{T}_{1}(u)=\mathbf{R}_{v}(u, 0), \mathbf{T}_{3}(u)=\mathbf{R}_{v}(u, 1), \mathbf{T}_{2}(v)=\mathbf{R}_{u}(0, v), \mathbf{T}_{4}(v)=\mathbf{R}_{u}(1, v),
$$

and $\mathbf{T}_{i}(t)$ should lie in the tangent plane which is spanned by $\mathbf{r}_{i}^{\prime}(t)$ and $\left[\mathbf{r}_{i}^{\prime}(t) \times \mathbf{r}_{i}^{\prime \prime}(t)\right] \times \mathbf{r}_{i}^{\prime}(t)$ at the point $\mathbf{r}_{i}(t)$. Then they can be expressed as

$$
\mathbf{T}_{i}(t)=x_{i}(t) \mathbf{r}_{i}^{\prime}(t)+y_{i}(t)\left[\mathbf{r}_{i}^{\prime}(t) \times \mathbf{r}_{i}^{\prime \prime}(t)\right] \times \mathbf{r}_{i}^{\prime}(t), t \in[0,1]
$$

where $x_{i}(t)=\sum_{j=0}^{5} \alpha_{i j} N_{j, 3}(t)$ and $y_{i}(t)=\sum_{j=0}^{1} \beta_{i j} N_{j, 1}(t)$ are scalar functions over the knot vector $\mathbf{U}_{i}=\left\{0,0,0,0, u_{i 4}, u_{i 5}, 1,1,1,1\right\}$ and $\mathbf{V}=\{0,0,1,1\}$, respectively.

\subsection{Identification of transverse tangent vectors}

The coefficients $\alpha_{i j}$ and $\beta_{i j}$ of $\mathbf{T}_{i}(t)(i=1, \cdots, 4)$ are real and can be obtained from the following conditions:

- Interpolation of the tangent vectors,

$$
\begin{aligned}
& \mathbf{T}_{1}(0)=\mathbf{r}_{2}^{\prime}(0), \mathbf{T}_{1}(1)=\mathbf{r}_{4}^{\prime}(0), \mathbf{T}_{2}(0)=\mathbf{r}_{1}^{\prime}(0), \mathbf{T}_{2}(1)=\mathbf{r}_{3}^{\prime}(0), \\
& \mathbf{T}_{3}(0)=\mathbf{r}_{2}^{\prime}(1), \mathbf{T}_{3}(1)=\mathbf{r}_{4}^{\prime}(1), \mathbf{T}_{4}(0)=\mathbf{r}_{1}^{\prime}(1), \mathbf{T}_{4}(1)=\mathbf{r}_{3}^{\prime}(1) .
\end{aligned}
$$

$\mathbf{T}_{1}(0)=\mathbf{r}_{2}^{\prime}(0)$ is equivalent to

$$
\frac{\alpha_{10}}{u_{14}}\left(\Delta \mathbf{P}_{10}\right)+\frac{18 \beta_{10}}{u_{14}^{3} u_{15}}\left[\left(\Delta \mathbf{P}_{10}\right) \times\left(\Delta^{2} \mathbf{P}_{10}\right)\right] \times\left(\Delta \mathbf{P}_{10}\right)=\frac{1}{u_{24}}\left(\Delta \mathbf{P}_{20}\right) .
$$

Then $\alpha_{10}$ and $\beta_{10}$ can be represented as

$$
\alpha_{10}=\frac{u_{14}}{u_{24}} \cdot \frac{\left(\Delta \mathbf{P}_{10}\right) \cdot\left(\Delta \mathbf{P}_{20}\right)}{\left\|\Delta \mathbf{P}_{10}\right\|^{2}}, \beta_{10}=\frac{u_{14}^{3} u_{15}}{18 u_{24}} \cdot \frac{\operatorname{det}\left(\left(\Delta \mathbf{P}_{10}\right) \times\left(\Delta^{2} \mathbf{P}_{10}\right),\left(\Delta \mathbf{P}_{10}\right),\left(\Delta \mathbf{P}_{20}\right)\right)}{\left\|\left(\Delta \mathbf{P}_{10}\right) \times\left(\Delta^{2} \mathbf{P}_{10}\right) \times\left(\Delta \mathbf{P}_{10}\right)\right\|^{2}} .
$$

$\mathbf{T}_{1}(1)=\mathbf{r}_{4}^{\prime}(0)$ is equivalent to

$$
\frac{\alpha_{15}}{1-u_{15}}\left(\Delta \mathbf{P}_{14}\right)-\frac{18 \beta_{11}}{\left(1-u_{14}\right)\left(1-u_{15}\right)^{3}}\left[\left(\Delta \mathbf{P}_{14}\right) \times\left(\Delta^{2} \mathbf{P}_{13}\right)\right] \times\left(\Delta \mathbf{P}_{14}\right)=\frac{1}{u_{44}}\left(\Delta \mathbf{P}_{40}\right),
$$

then

$$
\alpha_{15}=\frac{1-u_{15}}{u_{44}} \cdot \frac{\left(\Delta \mathbf{P}_{14}\right) \cdot\left(\Delta \mathbf{P}_{40}\right)}{\left\|\Delta \mathbf{P}_{14}\right\|^{2}}, \beta_{11}=-\frac{\left(1-u_{14}\right)\left(1-u_{15}\right)^{3}}{18 u_{44}} \cdot \frac{\left.\operatorname{det}\left(\left(\Delta \mathbf{P}_{14}\right) \times\left(\Delta^{2} \mathbf{P}_{13}\right)\right],\left(\Delta \mathbf{P}_{14}\right),\left(\Delta \mathbf{P}_{40}\right)\right)}{\left.\|\left(\Delta \mathbf{P}_{14}\right) \times\left(\Delta^{2} \mathbf{P}_{13}\right)\right] \times\left(\Delta \mathbf{P}_{14}\right) \|^{2}} .
$$

Similarly, the first and the last coefficients of $x_{i}(t)$ and $y_{i}(t), i=2, \cdots, 4$, can be solved. 
- Compatibility of the twist vectors,

$$
\begin{aligned}
& \mathbf{R}_{u, v}(0,0):=\mathbf{T}_{1}^{\prime}(0)=\mathbf{T}_{2}^{\prime}(0), \mathbf{R}_{u, v}(0,1):=\mathbf{T}_{2}^{\prime}(1)=\mathbf{T}_{3}^{\prime}(0), \\
& \mathbf{R}_{u, v}(1,0):=\mathbf{T}_{1}^{\prime}(1)=\mathbf{T}_{4}^{\prime}(0), \mathbf{R}_{u, v}(1,1):=\mathbf{T}_{3}^{\prime}(1)=\mathbf{T}_{4}^{\prime}(1) .
\end{aligned}
$$

Each equation above leads to two pairs of equations from corresponding coordinates, such that $\alpha_{i 1}$ and $\alpha_{i 4}, i=1, \cdots, 4$, can be obtained. Additionally, if the angle between $\mathbf{r}_{1}^{\prime}(0)$ and $\mathbf{r}_{2}^{\prime}(0)$ at the corner $\mathbf{P}_{10}$ is $A_{12}$, projecting $\mathbf{T}_{1}^{\prime}(0)$ and $\mathbf{T}_{2}^{\prime}(0)$ on $\mathbf{N}_{12}$, we have

$$
\mathbf{T}_{1}^{\prime}(0) \cdot \mathbf{N}_{12}=\tau_{1}(0)\left\|\mathbf{r}_{1}^{\prime}(0)\right\|\left\|\mathbf{r}_{2}^{\prime}(0)\right\| \sin A_{12}, \quad \mathbf{T}_{2}^{\prime}(0) \cdot \mathbf{N}_{12}=-\tau_{2}(0)\left\|\mathbf{r}_{1}^{\prime}(0)\right\|\left\|\mathbf{r}_{2}^{\prime}(0)\right\| \sin A_{12}
$$

Obviously, $\mathbf{T}_{1}^{\prime}(0) \cdot \mathbf{N}_{12}=\mathbf{T}_{2}^{\prime}(0) \cdot \mathbf{N}_{12}$ if and only if $\tau_{1}(0)=-\tau_{2}(0)$, which satisfies the constraint $(C 3)$. Other relations can be derived at the other corners in the same way.

The residual coefficients $\alpha_{i j}=0(i=1, \cdots, 4, j=2,3)$ can be freely chosen, then $\mathbf{T}_{i}(t)$ are determined.

\subsection{B-spline surface construction}

It's necessary to represent $\mathbf{T}_{i}(t)$ as B-spline form according to the expression of B-spline surface interpolating Bspline curves. Since the degree of $\left[\mathbf{r}^{\prime}(t) \times \mathbf{r}^{\prime \prime}(t)\right] \times \mathbf{r}^{\prime}(t)$ is quartic, the degree of $\mathbf{T}_{i}(t)$ is assumed to be at least quintic. In order to ensure the interpolating surface of low degree, we let the degree of $\mathbf{T}_{i}(t)$ be quintic. Then

$$
\mathbf{T}_{i}(t)=x_{i}(t) \mathbf{r}_{i}^{\prime}(t)+y_{i}(t)\left[\mathbf{r}_{i}^{\prime}(t) \times \mathbf{r}_{i}^{\prime \prime}(t)\right] \times \mathbf{r}_{i}^{\prime}(t)=\sum_{k=0}^{13} \mathbf{E}_{k}^{i} N_{k, 5}(t)+\sum_{k=0}^{13} \mathbf{F}_{k}^{i} N_{k, 5}(t)=\sum_{k=0}^{13}\left(\mathbf{E}_{k}^{i}+\mathbf{F}_{k}^{i}\right) N_{k, 5}(t), i=1, \cdots, 4,
$$

where $\mathbf{E}_{k}^{i}$ and $\mathbf{F}_{k}^{i}$ correspond to fourteen control points of B-spline functions and can be determined in the following.

Taking the compatibility of the interpolation condition Eq. (11) along the boundaries into consideration, we choose that the degree of tensor-product B-spline surface $\mathbf{R}(u, v)$ is bi-quintic, that is

$$
\mathbf{R}(u, v)=\sum_{i=0}^{13} \sum_{j=0}^{13} \mathbf{Q}_{i, j} N_{i, 5}(u) N_{j, 5}(v),
$$

where $\mathbf{Q}_{i, j}, i, j=0, \cdots, 13$, are the control points of the surface.

Let $\Delta \mathbf{P}_{i j_{1}}=\frac{\mathbf{P}_{i j_{1}+1}-\mathbf{P}_{i j_{1}}}{u_{i j_{1}+4}-u_{i} j_{1}+1}, \Delta^{2} \mathbf{P}_{i j_{1}}=\frac{\Delta \mathbf{P}_{i j_{1}+1}-\Delta \mathbf{P}_{i j_{1}}}{u_{i j_{1}+4}-u_{i} j_{1}+2}$ and $N_{k, p}(t)(p=1, \cdots, 5)$ be defined over knot vectors

$$
\mathbf{U}_{p}^{i}=\{\underbrace{0, \cdots, 0}_{p+1}, \underbrace{u_{i 4}, \cdots, u_{i 4}}_{p-1}, \underbrace{u_{i 5}, \cdots, u_{i 5}}_{p-1}, \underbrace{1, \cdots, 1}_{p+1}\}:=\left\{t_{0}^{i}, \cdots, t_{4 p-1}^{i}\right\} .
$$

The quintic B-spline functions defined on above knot vectors have 14 control points. That's why the B-spline surface has $(13+1) \times(13+1)$ control points. Based on the product formula of B-spline (Mørken, 1991), we have

$$
x_{i}(t) \mathbf{r}_{i}^{\prime}(t)=\sum_{k=0}^{13} \mathbf{E}_{k}^{i} N_{k, 5}(t)
$$

where $\mathbf{E}_{k}^{i}=\frac{3}{10} \sum_{P^{2} \in \Pi^{5}} \sum_{j_{1}} \sum_{j} \alpha_{i j} \cdot \Delta \mathbf{P}_{i j_{1}} \cdot \alpha_{j, 3, \mathbf{U}_{3}^{i}, \mathbf{t}_{Q^{3}}^{i}}(k) \cdot \alpha_{j_{1}, 2, \mathbf{U}_{2}^{i}, \mathbf{t}_{P^{2}}^{i}}(k)$, and

$$
\mathbf{t}_{P^{2}}^{i}=\left\{\cdots, t_{k}^{i}, t_{k+p_{1}}^{i}, t_{k+p_{2}}^{i}, t_{k+6}^{i}, \cdots\right\}, \mathbf{t}_{Q^{3}}^{i}=\left\{\cdots, t_{k}^{i}, t_{k+q_{1}}^{i}, t_{k+q_{2}}^{i}, t_{k+q_{3}}^{i}, t_{k+6}^{i}, \cdots\right\} .
$$

Here $\alpha_{j_{1}, m, \mathbf{U}_{m}^{i}, \mathbf{t}_{p m}^{i}}(k)$ and $\alpha_{j, n-m, \mathbf{U}_{n-m}^{i}, \mathbf{t}_{Q^{n-m}}^{i}}(k)$ are discrete B-splines referred to Definition 2. Element $p_{i}(i=1, \cdots, m)$ of $P^{m}=\left\{p_{1}, p_{2}, \cdots, p_{m}\right\}$ is chosen from $I^{n}=\{1,2, \cdots, n\}$, while $Q^{n-m}=\left\{q_{1}, q_{2}, \cdots, q_{n-m}\right\}=I^{n}-P^{m}, \Pi^{n}=\bigcup_{p_{i} \in I^{n}} P^{m}$.

Similarly, we have

$$
\mathbf{r}_{i}^{\prime}(t) \times \mathbf{r}_{i}^{\prime \prime}(t)=\sum_{k=0}^{7} \mathbf{G}_{k}^{i} N_{k, 3}(t),
$$

where $\mathbf{G}_{k}^{i}=6 \sum_{P^{1} \in \Pi^{3}} \sum_{j_{1}} \sum_{j_{2}} \Delta \mathbf{P}_{i j_{1}} \times \Delta^{2} \mathbf{P}_{i j_{2}} \cdot \alpha_{j_{1}, 2, \mathbf{U}_{2}^{i}, \mathbf{t}_{Q^{2}}^{i}}(k) \cdot \alpha_{j_{2}, 1, \mathbf{U}_{1}^{i}, \mathbf{t}_{P 1}^{i}}(k)$.

Notice that $\mathbf{r}_{i}^{\prime}(t) \times \mathbf{r}_{i}^{\prime \prime}(t)$ is actually quadratic, then

$$
\mathbf{r}_{i}^{\prime}(t) \times \mathbf{r}_{i}^{\prime \prime}(t)=\sum_{k=0}^{4} \mathbf{H}_{k}^{i} N_{k, 2}(t),
$$


where $\mathbf{H}_{k}^{i}=\mathbf{P}_{k}^{0}(k=0, \cdots, 4), \mathbf{P}_{k}^{2}=\mathbf{G}_{k}^{i}(k=0, \cdots, 7), l_{2}=7, l_{j-1}=l_{j}-2, j=1,2$,

$$
\mathbf{P}_{k}^{j-1}=\left\{\begin{array}{l}
\mathbf{P}_{k}^{j}, 0 \leq k \leq l_{j}-3, \\
\frac{\mathbf{P}_{k}^{j}}{1-a_{a, 2}^{j}}-\frac{a_{k, 2}^{j} \mathbf{P}_{k-1}^{j-1}}{1-a_{k, 2}^{j}}, l_{j}-2 \leq k \leq l_{j}-1, \\
\mathbf{P}_{k+1}^{j}, l_{j} \leq k \leq 4+j,
\end{array}\right.
$$

and $a_{k, 2}^{j}$ is defined in (Wang and Deng, 2007).

Continue to use the product formula of B-spline (Mørken, 1991), we get

$$
\mathbf{r}_{i}^{\prime}(t) \times \mathbf{r}_{i}^{\prime \prime}(t) \times \mathbf{r}_{i}^{\prime}(t)=\sum_{k=0}^{10} \mathbf{L}_{k}^{i} N_{k, 4}(t), y_{i}(t) \mathbf{r}_{i}^{\prime}(t) \times \mathbf{r}_{i}^{\prime \prime}(t) \times \mathbf{r}_{i}^{\prime}(t)=\sum_{k=0}^{13} \mathbf{F}_{k}^{i} N_{k, 5}(t),
$$

where $\mathbf{L}_{k}^{i}=\frac{1}{2} \sum_{P^{2} \in \Pi^{4}} \sum_{j_{1}} \sum_{j_{3}} \mathbf{H}_{j_{1}}^{i} \times \Delta \mathbf{P}_{i j_{3}} \cdot \alpha_{j_{1}, 2, \mathbf{U}_{2}^{i}, \mathbf{t}_{p^{2}}^{i}}(k) \cdot \alpha_{j_{3}, 2, \mathbf{U}_{2}^{i}, \mathbf{t}_{Q^{2}}^{i}}(k), \mathbf{F}_{k}^{i}=\frac{1}{5} \sum_{P_{1} \in \Pi^{5}} \sum_{j_{2}} \sum_{j_{4}} \beta_{i} j_{j_{2}} \cdot \mathbf{L}_{j_{4}}^{i} \cdot \alpha_{j_{2}, 1, \mathbf{U}_{1}^{i}, \mathbf{t}_{p 1}^{i}}(k) \cdot \alpha_{j_{4}, 4, \mathbf{U}_{4}, \mathbf{t}_{Q^{4}}^{i}}(k)$.

By the degree elevation formula of B-spline curve (Wang and Deng, 2007), the boundary cubic B-spline curve can be elevated to the quintic as

$$
\mathbf{r}_{i}(t)=\sum_{j=0}^{13} \mathbf{M}_{j}^{i} N_{j, 5}(t)
$$

such that the outermost control points $\mathbf{Q}_{0, i}, \mathbf{Q}_{i, 0}, \mathbf{Q}_{13, i}, \mathbf{Q}_{i, 13}$ can be determined by the boundary control points $\mathbf{M}_{j}^{i}$. Namely,

$$
\mathbf{Q}_{0, i}=\mathbf{M}_{i}^{1}, \mathbf{Q}_{i, 0}=\mathbf{M}_{i}^{2}, \mathbf{Q}_{13, i}=\mathbf{M}_{i}^{3}, \mathbf{Q}_{i, 13}=\mathbf{M}_{i}^{4}, i=0, \cdots, 13 .
$$

From Eq. (13) and Eq. (14), we can get $(i=1, \cdots, 12)$

$$
\begin{aligned}
& \mathbf{Q}_{1, i}=\mathbf{M}_{i}^{1}+\frac{u_{14}}{5}\left(\mathbf{E}_{i}^{1}+\mathbf{F}_{i}^{1}\right), \mathbf{Q}_{12, i}=\mathbf{M}_{i}^{3}-\frac{1-u_{35}}{5}\left(\mathbf{E}_{i}^{3}+\mathbf{F}_{i}^{3}\right), \\
& \mathbf{Q}_{i, 1}=\mathbf{M}_{i}^{2}+\frac{u_{22}}{5}\left(\mathbf{E}_{i}^{2}+\mathbf{F}_{i}^{2}\right), \mathbf{Q}_{i, 12}=\mathbf{M}_{i}^{4}-\frac{1-u_{45}}{5}\left(\mathbf{E}_{i}^{4}+\mathbf{F}_{i}^{4}\right),
\end{aligned}
$$

which is compatible at the corner points. It's easy to see that the vectors $\mathbf{M}_{i}^{1} \mathbf{Q}_{1, i}$ are parallel to the vectors $\mathbf{O}\left(\mathbf{E}_{i}^{1}+\mathbf{F}_{i}^{1}\right)$, where $\mathbf{O}$ is the origin, then the geometric relations between them refer to Fig. 2. The other two arrays of control points along the other three boundaries have the similar geometric relations.

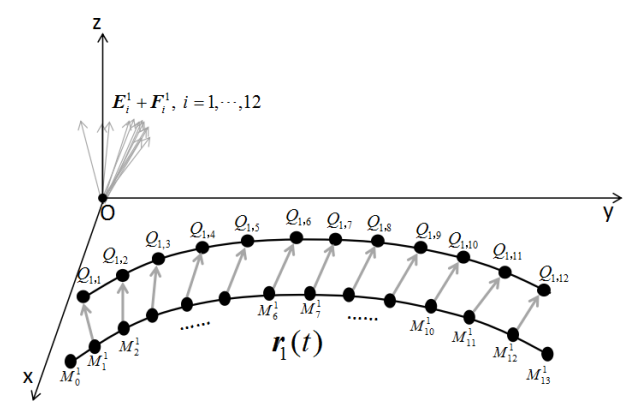

Fig. 2 The geometric relations of two arrays of control points along $\mathbf{r}_{1}(t)$.

Notice that, two arrays of control points along the boundary curves have been obtained. The rest control points of the surface can be freely chosen or obtained by minimizing the thin plate spline energy

$$
\int_{0}^{1} \int_{0}^{1}\left(\left\|\mathbf{R}_{u u}(u, v)\right\|^{2}+2\left\|\mathbf{R}_{u v}(u, v)\right\|^{2}+\left\|\mathbf{R}_{v v}(u, v)\right\|^{2}\right) d u d v .
$$

\section{Examples}

Example 1. For cubic B-spline boundary curves represented as Eq. (8), two arrays of control points of the resulting $B$-spline surface are obtained by Eq. (15) and Eq. (16), while the other control points of the surface are obtained from Eq. (17). In the following examples, the control points of the surfaces are determined in the same way. Figure 3 shows two pairs of B-spline surface of bi-quintic interpolating the cubic B-spline asymptotic quadrilateral. 


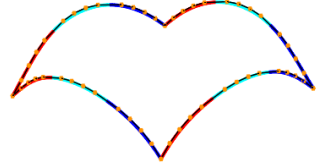

(a) Quadrilateral and control points.

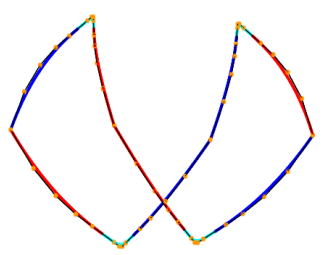

(d) Quadrilateral and control points.

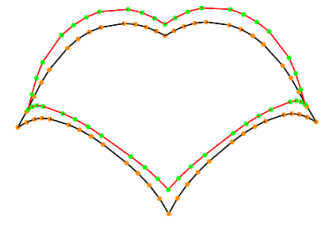

(b) Two arrays of control points.

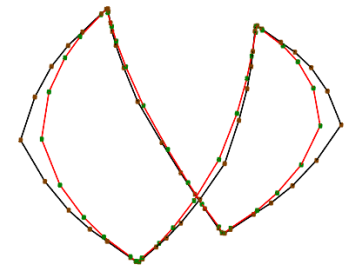

(e) Two arrays of control points.

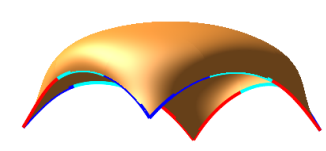

(c) The resulting B-spline surface.

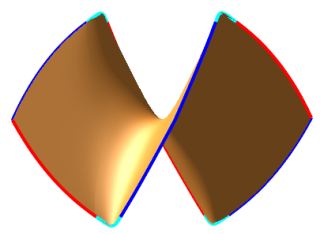

(f) The resulting B-spline surface.

Fig. 3 B-spline surface interpolating the B-spline asymptotic quadrilateral.

Example 2. A curve satisfying $\boldsymbol{r}^{\prime \prime}=0$, that is the curve with zero curvature, is a line, which is also an asymptotic curve from Lemma 1. For $\boldsymbol{r}_{1}^{\prime \prime}=0$ and $\boldsymbol{r}_{i}^{\prime \prime} \neq 0, i=2,3,4$, based on the analysis in Section 3.2, we can construct a surface bounded by a quadrilateral with one zero curvature. To be compatible with the construction process, some conditions are modified as $k_{1}(0)=0, k_{1}(1)=0, \lambda_{13}=0, \mu_{12}=0, \tau_{2}(0)=0, \tau_{4}(0)=0$. And the definition of the tangent vector $\boldsymbol{T}_{1}(t)$ is changed as

$$
\boldsymbol{T}_{1}(t)=x_{1}(t) \boldsymbol{r}_{1}^{\prime}(t)+y_{1}(t) \boldsymbol{r}_{2}^{\prime}(t)+z_{1}(t) \boldsymbol{r}_{4}^{\prime}(1-t), t \in[0,1],
$$

where $x_{1}(t)=\sum_{j=0}^{5} \alpha_{1 j} N_{j, 3}(t), y_{1}(t)=\sum_{j=0}^{5} \beta_{1 j} N_{j, 3}(t), z_{1}(t)=\sum_{j=0}^{5} \delta_{1 j} N_{j, 3}(t)$ satisfying $x_{1}(0)=0, y_{1}(0)=1, z_{1}(0)=0$ and $x_{1}(1)=0, y_{1}(1)=0, z_{1}(1)=1$. Similar to the construction in Example 1, we can get the resulting surface. Figure 4 shows two quintic B-spline surfaces interpolating the cubic B-spline asymptotic quadrilaterals with one linear boundary curve, where both $\boldsymbol{r}_{2}(t)$ and $\boldsymbol{r}_{4}(t)$ curve upwards and downwards, respectively.

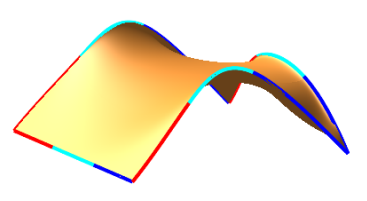

(a) $\mathbf{r}_{2}(t), \mathbf{r}_{4}(t)$ curve upwards.

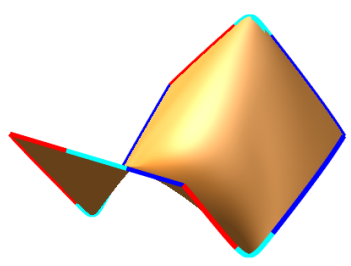

(b) $\mathbf{r}_{2}(t), \mathbf{r}_{4}(t)$ curve downwards.

Fig. 4 B-spline surface interpolating the B-spline asymptotic quadrilateral with one linear boundary curve.

Example 3. For two of the boundary curves with zero curvatures, we assume $\boldsymbol{r}_{1}^{\prime \prime}=0, \boldsymbol{r}_{2}^{\prime \prime}=0$ and $\boldsymbol{r}_{i}^{\prime \prime} \neq 0, i=3,4$. Besides the conditions related with $\boldsymbol{r}_{1}$ in Example 2, the conditions related with $\boldsymbol{r}_{2}$ can be set as $k_{2}(0)=0, k_{2}(1)=0$, $\lambda_{22}=0, \mu_{23}=0, \tau_{1}(0)=0, \tau_{3}(0)=0, \boldsymbol{T}_{2}(t)=x_{2}(t) \boldsymbol{r}_{2}^{\prime}(t)+y_{2}(t) \boldsymbol{r}_{1}^{\prime}(t)+z_{2}(t) \boldsymbol{r}_{3}^{\prime}(1-t)$, and $x_{2}(t), y_{2}(t), z_{2}(t)$ are cubic $B$-splines. Figure 5-(a), (b) illustrate two $B$-spline surfaces interpolating the $B$-spline asymptotic quadrilaterals with two intersecting lines. Similarly, we can construct the B-spline surface interpolating the B-spline asymptotic quadrilaterals with two opposite lines as shown in Fig. 5-(c), (d).

Example 4. By the method presented in above examples, the B-spline surfaces interpolating quadrilateral with three lines can be constructed as shown in Fig. 6. 


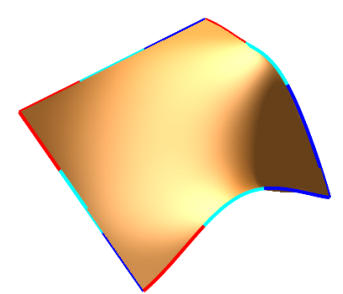

(a) $\mathbf{r}_{3}(t)$ curves upwards.

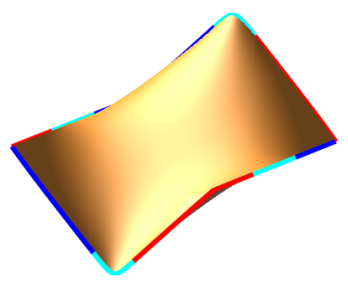

(c) $\mathbf{r}_{2}(t)$ curves upwards.

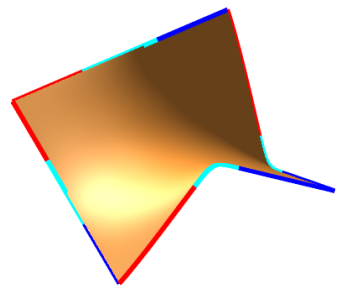

(b) $\mathbf{r}_{3}(t)$ curves downwards.

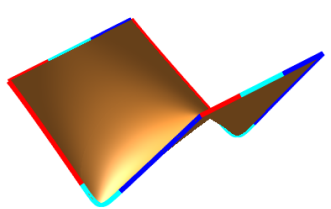

(d) $\mathbf{r}_{2}(t)$ curves downwards.

Fig. 5 B-spline surface interpolating the B-spline asymptotic quadrilateral with two linear boundary curves.

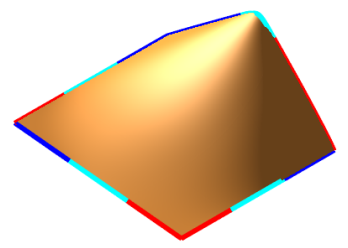

(a) $\mathbf{r}_{4}(t)$ curves upwards.

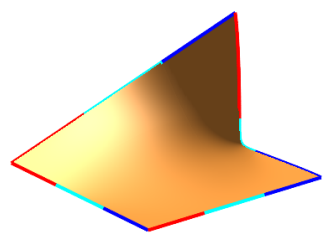

(b) $\mathbf{r}_{4}(t)$ curves downwards.

Fig. 6 B-spline surface interpolating the B-spline asymptotic quadrilateral with three linear boundary curves.

\section{Boundary curves with inflection points}

If $\xi$ is an inflection of curve $\mathbf{r}(t)$, then $\mathbf{r}^{\prime}(\xi) \times \mathbf{r}^{\prime \prime}(\xi)=0$, and the left and the right limits of the binormal vector $\mathbf{b}(t)$ are anti-parallel. In fact, if each of the boundary curves $\mathbf{r}_{i}(t), i=1, \ldots, 4$, has one or two inflections, then the total number of reversals of the binormal vectors $\mathbf{b}(t)$ along the boundary curves is still even. The cubic B-spline quadrilateral satisfying the constraints $(C 1)-(C 3)$ with inflections can be constructed as the boundary curves of B-spline surface, based on the previous analysis. The detail will be omitted and we show the resulting surfaces by two examples as follows.

Example 5. Let $\boldsymbol{r}_{i}(t), i=1, \ldots, 4$, be orthogonal at four corner points and each of them has only one inflection. The bi-quintic B-spline surface interpolates the B-spline asymptotic quadrilateral with these inflections as shown in Fig. 7.

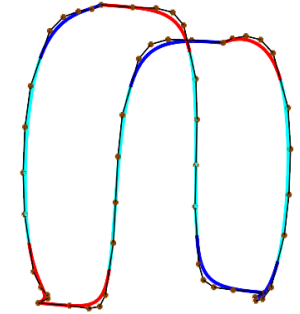

(a) Each boundary curve with one infection.

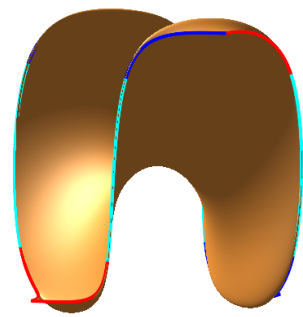

(b) The resulting B-spline surface.

Fig. 7 B-spline surface interpolating the B-spline asymptotic quadrilateral with inflections. 
Example 6. Each curve of $\boldsymbol{r}_{i}(t), i=1, \ldots, 4$, has two inflection points. Figure 8 shows the bi-quintic B-spline surface interpolating the B-spline asymptotic quadrilateral.

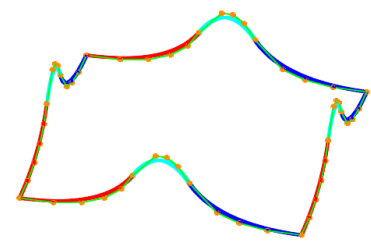

(a) Each boundary curve with two infections.

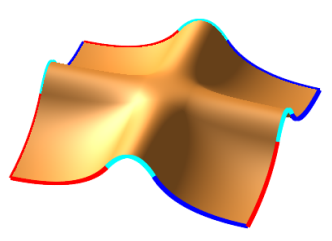

(b) The resulting B-spline surface.

Fig. 8 B-spline surface interpolating the B-spline asymptotic quadrilateral with inflections.

\section{Conclusions}

In the paper, we generalize the results on surfaces interpolating one or two curves as the asymptotes to construct surface interpolating quadrilateral as its asymptotic boundary curves. For the given corner data including positions, tangents and curvatures of a B-spline quadrilateral, we analyze the constraint conditions for the boundary curves to be asymptotic curves. Similar to the interpolated geodesic or lines of curvature quadrilateral, the identify constraints on boundary quadrilateral are required to be satisfied for the surface interpolating the asymptotic quadrilateral. After identifying optimized asymptotic quadrilateral, a smooth B-spline surface with minimized thin plate spline energy is constructed to interpolate them. For the cubic B-spline boundary quadrilateral, the degree of a tensor-product B-spline surface is bi-quintic. The presented method is illustrated by several examples including the condition that the boundary quadrilateral with zero curvatures. Interpolating asymptotic quadrilaterals with inflections is also discussed in the paper and two examples show the resulting tensor-product B-spline surfaces. Be compatible with commercial CAD systems, the surfaces constructed by above method are smooth and of low degrees. The experimental results show good agreement with the theoretical analysis.

The proposed construction model is realized by the B-spline surface, which is more flexible than the ruled surfaces in $\mathrm{CAD}$ model for architecture and mechanical design. And the surface interpolating the closed boundary curve is more interesting for surface modeling in architecture and mechanical design. Moreover, some present researches also focused on the applications of the asymptotic curve of surface in architecture, such as (Flory and Pottmann, 2010). These facts indicate the potential applications of the proposed method in architecture, architectural geometry, mechanical design or other fields. We will study on them in our future work.

\section{Acknowledgements}

This work is partly supported by the National Natural Science Foundation of China (Nos. 11671068, 11271060, 11401077), Fundamental Research of Civil Aircraft (No. MJ-F-2012-04), and the Fundamental Research Funds for the Central Universities (No. DUT16LK38, DUT17LK15).

\section{References}

Angenent, S. B. and Velázquez, J. J. L., Asymptotic shape of cusp singularities in curve shortening, Duke Mathematical Journal, Vol.77, No.1 (1995), pp. 71-110.

Atalay, G. S. and Kasap, E., Surfaces family with common null asymptotic, Applied Mathematics and Computation, Vol.260, No.C (2015), pp. 135-139.

Atalay, G. S. and Kasap, E., Surfaces family with common smarandache asymptotic curve according to bishop frame in Euclidean 3-space, Boletim Da Sociedade Paranaense De Matematica, Vol.34, No.1 (2016), pp. 187-202.

Bayram, E., Güler, F. and Kasap, E., Parametric representation of a surface pencil with a common asymptotic curve, Computer-Aided Design, Vol.44, No.7 (2012), pp. 637-643. 
Bayram, E., Ergün, E. and Kasap, E., Surface family with a common natural asymptotic lift, Journal of Science and Arts, Vol.2, No.31 (2015), pp. 117-124.

Bayram, E. and Bilici, M., Surface family with a common involute asymptotic curve, International Journal of Geometric Methods in Modern Physics, Vol.13, No.5 (2016), pp. 621-629.

Biard, L., Farouki, R. T. and Szafran, N., Construction of rational surface patches bounded by lines of curvature, Computer Aided Geometric Design, Vol.27, No.5 (2010), pp. 359-371.

Chen, W. H., Differential Geometry, 5th edition(2011), Peking University Press. (In Chinese)

Contopoulos, G., Asymptotic curve and escapes in Hamiltonian systems, Astronomy and Astrophysics, Vol.231, No.1 (1990), pp. 41-55.

Do Carmo, M. P., Differential Geometry of Curve and Surface(1976), Prentice Hall.

Farouki, R. T., Szafran, N. and Biard, L., Existence conditions for Coons patches interpolating geodesic boundary curves, Computer Aided Geometric Design, Vol.26, No.5 (2009a), pp. 599-614.

Farouki, R. T., Szafran, N. and Biard, L., Construction and smoothing of triangular Coons patches with geodesic boundary curves, Computer Aided Geometric Design, Vol.27, No.4 (2010), pp. 301-312.

Farouki, R. T., Szafran, N. and Biard, L., Construction of Bézier surface patches with Bézier curves as geodesic boundaries, Computer-Aided Design, Vol.41, No.11 (2009b), pp. 772-781.

Flöry, S. and Pottmann, H., Ruled Surfaces for Rationalization and Design in Architecture, Proceedings of the 30th Annual Conference of the Association for Computer Aided Design in Architecture (2010), pp. 103-109.

Hartman, P. and Winter, A., On the asymptotic curves of a surface, American Journal of Mathematics, Vol.73, No.1 (1951), pp. 149-172.

Kasap, E., Akyyildiz, F. T. and Orbay, K., A generalization of surface family with common spatial geodesic, Applied Mathematics and Computation, Vol.201, No.1 (2008), pp. 781-789.

Kitagawa, Y., Periodicity of the asymptotic curves on flat tori in $S^{3}$, Journal of the Mathematical Society of Japan, Vol.40, No.3 (1988) pp. 457-476.

Lane, E. P. and MacQueen, M. L., Asymptotic curve on a surface, Advances in Mathematics, Vol.61, No.3 (1986), pp. 640-648.

Li, C. Y., Wang, R. H. and Zhu, C. G., Parametric representation of a surface pencil with a common line of curvature, Computer-Aided Design, Vol.43, No.9 (2011a), pp. 1110-1117.

Li, C. Y., Wang, R. H. and Zhu, C. G., Design and $G^{1}$ connection of developable surface through Bézier geodesics, Applied Mathematics and Computation, Vol.218, No.7 (2011b), pp. 3199-3208.

Li, C. Y., Wang, R. H. and Zhu, C. G., A generalization of surface family with common line of curvature, Applied Mathematics and Computation, Vol.219, No.17 (2013a), pp. 9500-9507.

Li, C. Y., Wang, R. H. and Zhu, C. G., An approach for designing a developable surface through a given line of curvature, Computer-Aided Design, Vol.45, No.3 (2013b), pp. 621-627.

Liu, Y. and Wang, G. Z., Designing developable surface pencil through given curve as its common asymptotic curve, Journal of Zhejiang University(Engineering Science), Vol.47, No.7 (2013), pp. 1246-1252. (In Chinese)

Mørken, K., Some identities for products and degree raising of splines, Constructive Approximation, Vol.7, No.1 (1991), pp. 195-208.

Shi, L., Wang, J. and Pottmann, H., Smooth surfaces from rational bilinear patches, Computer Aided Geometric Design, Vol.31, No.1 (2014), pp. 1-12.

Wang, G. J., Tang, K. and Tai, C. L., Parametric representation of a surface pencil with a common spatial geodesic, Computer-Aided Design, Vol.36, No.5 (2004), pp. 447-459.

Wang, G. Z. and Deng, C. Y., On the degree elevation of B-spline curves and corner cutting, Computer Aided Geometric Design, Vol.24, No.2 (2007), pp. 90-98.

Wang, H. J. and Ni, Q., A new method of moving asymptotes for large-scale unconstrained optimization, Applied Mathematics and Computation, Vol.203, No.1 (2008), pp. 62-71.

Yang, H. G. and Wang, G. Z., Optimized design of Bézier surface through Bézier geodesic quadrilateral, Journal of Computational and Applied Mathematics, Vol.273, No.1 (2015a), pp. 264-273.

Yang, H. G. and Wang, G. Z., Construction of B-spline surface with B-spline curves as boundary geodesic quadrilateral, Journal of Computational and Applied Mathematics, Vol.290, No.C (2015b), pp. 104-113.

Yüzbasi, Z. K., On a family of surfaces with common asymptotic curve in the Galilean space $G_{3}$, Journal of Nonlinear Science and Applications, Vol.9, No.2 (2016), pp. 518-523. 